This is the peer reviewed version of the following article:

Karuthedath, S., Gorenflot, J., Firdaus, Y., Chaturvedi, N., De Castro, C. S. P., Harrison, G. T., et al. (2021). Intrinsic efficiency limits in low-bandgap non-fullerene acceptor organic solar cells. Nature Materials, 20, 378-384. doi:10.1038/s41563-020-00835-x.

, which has been published in final form at: 10.1038/s41563-020-00835-x

\title{
Intrinsic efficiency limits in low-bandgap non- fullerene acceptor organic solar cells
}

Karuthedath, S., Gorenflot, J., Firdaus, Y., Chaturvedi, N., De Castro, C. S. P., Harrison, G. T., et al. 


\section{Intrinsic Efficiency Limits in Low-bandgap Non-fullerene Acceptor Organic Solar Cells}

Safakath Karuthedath, ${ }^{1, \neq}$ Julien Gorenflot, ${ }^{1, \neq}$ Yuliar Firdaus, ${ }^{1}$ Neha Chaturvedi, ${ }^{1}$ Catherine S. P. De Castro, ${ }_{1}^{1}$ George T. Harrison, ${ }^{1}$ Jafar I. Khan, ${ }^{1}$ Anastasia Markina, ${ }^{2}$ Ahmed H. Balawi, ${ }^{1}$ Top Archie Dela Peña, ${ }^{1}$ Wenlan Liu, ${ }^{2}$ Ru-Ze Liang, ${ }^{1}$ Anirudh Sharma, ${ }^{1}$ Sri H. K. Paleti, ${ }^{1}$ Weimin Zhang, ${ }^{1}$ Yuanbao Lin, ${ }^{1}$ Erkki Alarousu, ${ }^{1}$ Dalaver H. Anjum, ${ }^{3}$ Pierre M. Beaujuge, ${ }^{1}$ Stefaan De Wolf, ${ }^{1}$ Iain McCulloch, ${ }^{1}$ Thomas D. Anthopoulos, ${ }^{1}$ Derya Baran, ${ }^{1}$ Denis Andrienko, ${ }^{2, *}$ and Frédéric Laquai ${ }^{1, *}$

${ }^{1}$ King Abdullah University of Science and Technology (KAUST), KAUST Solar Center (KSC), Physical Sciences and Engineering Division (PSE), Material Science and Engineering Program (MSE), Thuwal 23955-6900, Kingdom of Saudi Arabia

${ }^{2}$ Max Planck Institute for Polymer Research, Ackermannweg 10, D-55128 Mainz, Germany

${ }^{3}$ King Abdullah University of Science and Technology (KAUST), Imaging and Characterization Core Lab, 23955-6900 Thuwal, Kingdom of Saudi Arabia

${ }^{\ddagger}$ authors contributed equally

Corresponding authors: denis.andrienko@mpip-mainz.mpg; frederic.laquai@kaust.edu.sa;

Keywords: organic solar cells, non-fullerene acceptors, energy transfer, charge transfer, driving force

In bulk heterojunction (BHJ) organic solar cells (OSCs) both the electron affinity (EA) and ionization energy (IE) offsets at the donor-acceptor interface should equally control exciton dissociation. Here, we demonstrate that in low-bandgap non-fullerene acceptor (NFA) BHJs ultrafast donor-to-acceptor energy transfer precedes hole transfer from the acceptor to the donor and thus renders the EA offset virtually unimportant. Moreover, sizeable bulk IE offsets of about $0.5 \mathrm{eV}$ are needed for efficient charge generation and high internal quantum efficiencies, since energy level bending at the donor-NFA interface caused by the acceptors' quadrupole moments prevents efficient exciton-to-CT state conversion at low IE offsets. The same bending however, is the origin of the barrier-less CT state to free charge conversion. Our results provide a comprehensive picture of the photophysics of NFA-based blends, and convincingly prove that sizeable bulk IE offsets are essential to design efficient BHJ OSCs based on low-bandgap NFAs. 


\section{Introduction}

The minimum driving force required for efficient charge separation in bulk heterojunction (BHJ) organic solar cells (OSCs) has remained controversial. ${ }^{1-10}$ In BHJs with low-bandgap non-fullerene acceptors (NFAs) both the electron donor and acceptor harvest photons, preferably in complementary spectral regions. Consequently, two channels of charge generation are expected, electron transfer from the donor to the acceptor and hole transfer from the acceptor to the donor. In thin film BHJs, the former is driven by the electron affinity (EA) offset, the latter by the ionization energy (IE) offset.

In fullerene-based systems, the requirement of a minimal EA offset of around $0.3 \mathrm{eV}$ was experimentally identified ${ }^{11}$ and repeatedly confirmed. ${ }^{12-15}$ However, recent reports of efficient NFAbased BHJs imply that this requirement may not hold for NFAs. Indeed, a number of studies concluded that charge generation in NFA BHJs can be efficient even for zero offsets, contrary to fullerene-based systems. ${ }^{11,13-15}$ Moreover, negligible driving forces for charge separation via hole transfer $^{8,16-20}$ and electron transfer ${ }^{21-23}$ are often used as an explanation of low energy losses in highperforming NFA blends. ${ }^{24}$

To quantify the position of energy levels in BHJs, characterization techniques such as ultraviolet photoelectron spectroscopy (UPS), photoemission spectroscopy in air (PESA), and cyclic voltammetry $(\mathrm{CV})$ have been used. Notably, only $\mathrm{CV}$ supports the idea of zero $\mathrm{IE}^{8,16-20}$ and $\mathrm{EA}^{21-23}$ offsets. It is, however, known that $\mathrm{CV}$ does not provide reliable estimates of IEs and EAs of solid films. ${ }^{25}$ PM6:Y6 blend, for example, has zero offset according to $\mathrm{CV}$ and $0.5 \mathrm{eV}$ when measured by UPS. ${ }^{19}$ Apart from questioning the necessity for a type-II heterojunction in NFA BHJs, efficient charge generation with zero energy offsets implies that the mechanism of charge generation in NFAand fullerene-based blends are different. ${ }^{26}$ On the contrary, a few recent studies acknowledged that IE offsets might be important for charge generation, ${ }^{9,27,28}$ demonstrating an increase of the charge generation efficiency with the IE offset, however, typically only for a very limited number of structurally-related systems.

A coherent study providing uniform characterization of a large NFA series as well as a model explaining the origin of the IE offset dependence in NFA-based systems and the recently reported barrier-less charge separation in NFA blends ${ }^{29}$ is still missing, although it is a prerequisite for further optimization of energetic losses and internal quantum efficiency (IQE) of NFA BHJs. ${ }^{30}$

In this work, we demonstrate that the exciton-to-charge conversion efficiency (and, therefore, the IQE) of low-bandgap NFA-based BHJ solar cells increases with the IE offset between the donor and NFA, reaching its maximum for offsets of $\sim 0.5 \mathrm{eV}$. Surprisingly, charge generation does not depend 
on the EA offset. This is explained by ultrafast energy transfer from the donor to the acceptor. Indeed, by design, the photoluminescence (PL) of the higher bandgap donor overlaps with the absorption of the lower bandgap acceptor. This design principle that broadens the BHJ's photoactive spectral region and thereby increases the maximum available short circuit current density, facilitates Förster resonance energy transfer (FRET) from the donor to the NFA. ${ }^{31-34}$ Consequently, FRET becomes the major deactivation channel for donor excitons in NFA BHJs. In fullerene BHJs, FRET is limited by the low oscillator strength of fullerene molecules. ${ }^{35}$

Secondly, we demonstrate that bending of the energy levels at the donor-acceptor heterojunction, caused by the acceptors' quadrupole moments, increases the interfacial charge-transfer (CT) state energy. If this energy level bending is not counterbalanced by the IE offset, then it creates a barrier for exciton-to-CT state conversion. The same energy level bending however, leads to barrier-less CT state to free charge conversion energy, as recently observed experimentally. ${ }^{29,36}$ Finally yet importantly, the same energy level bending also explains the presence of energetically high-lying CT states reported for many NFA-based systems.

A straightforward implication of our study is a clear design principle for NFA materials: high excitonto-charge conversion yields can only be achieved for sizeable $(\sim 0.5 \mathrm{eV})$ bulk IE offsets, since only they provide sufficient driving force for dissociation of the excitons to CT states, and further to separated charge carriers.

\section{Results and discussion}

To reveal the impact of IE, EA, and FRET on charge generation in NFA BHJs, we studied a large set of representative donor-acceptor combinations: the small molecule electron donor DR3TBDTT (DR3), ${ }^{37}$ the widely-used donor polymers PTB7-Th (PCE10) and PBDBT-SF (PCE13), ${ }^{38}$ and the recently reported high-efficiency donor polymer PBDB-T-2F (PM6). ${ }^{39}$ Ten small molecule NFAs were selected as acceptors, including Y6, one of the current state-of-the-art NFAs, as well as PC ${ }_{71} \mathrm{BM}$ as reference. ${ }^{40}$ Chemical structures of all materials are shown in Fig. 1 alongside their IE and EA values, determined by ultraviolet photoemission spectroscopy (UPS), and inverted photoemission spectroscopy (IPES) respectively, as well as the average internal quantum efficiencies (IQEs) of respective BHJs (details of UPS, IPES can be found in Supplementary Note 1). ${ }^{41}$ Since IE offsets are essential in our study, we not only relied on UPS data, but also confirmed the materials' IEs by another technique, photoemission spectroscopy in air (PESA) (Supplementary Note 1). ${ }^{41,42}$ 

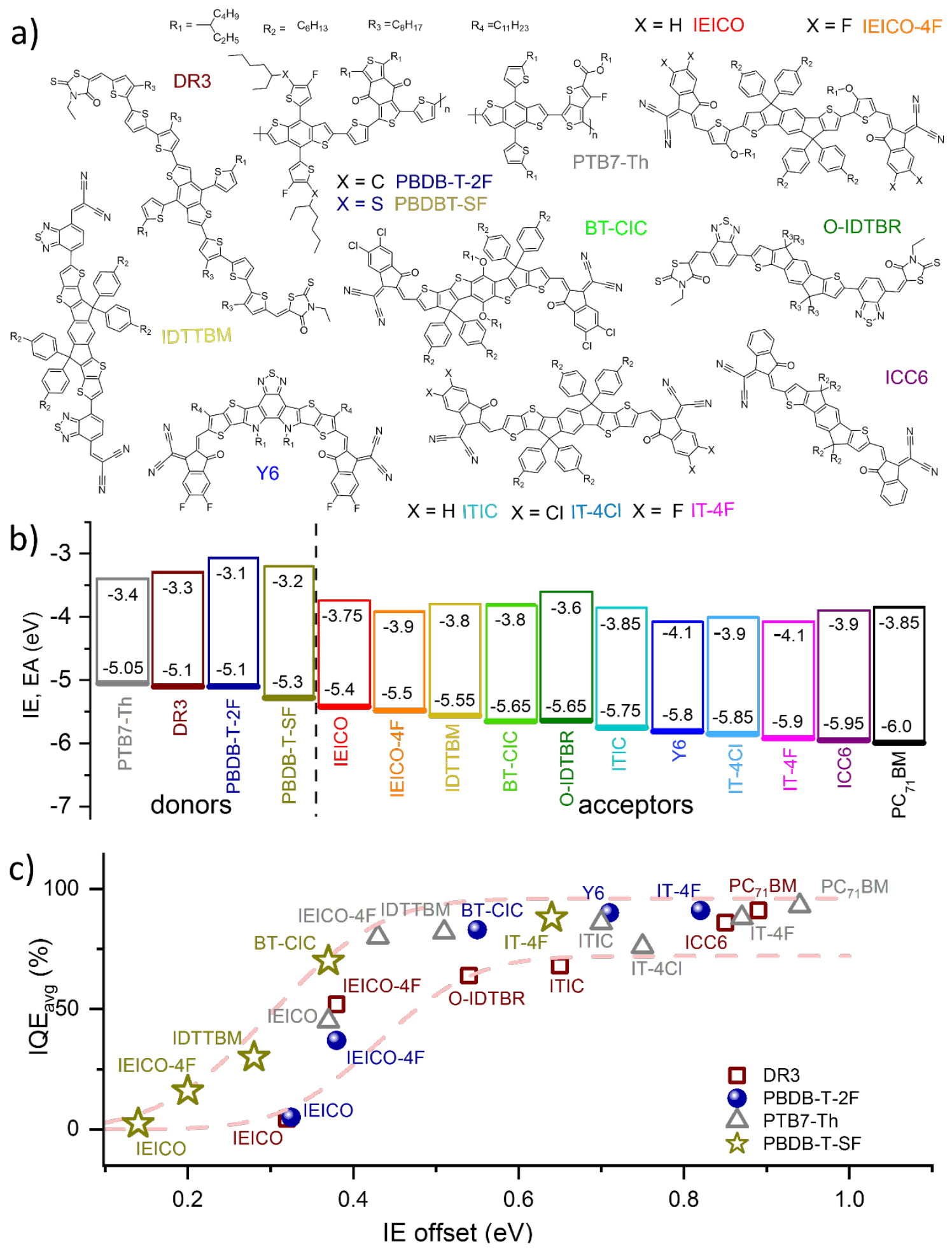

Fig. 1: Materials' structures, energy levels, and internal quantum efficiencies in photovoltaic devices. a) Chemical structures of the donor materials and non-fullerene acceptors investigated in this study. b) Ionization energy (IE) determined by ultraviolet photoelectron spectroscopy (UPS) and electron affinity (EA) by inverse photoemission spectroscopy (IPES). c) Spectrally averaged internal quantum efficiency (IQE avg $_{\text {) of optimized }}$ bulk heterojunction donor-NFA devices as a function of the IE offset between donor and acceptor for the small molecule donor DR3 and donor polymers PBDB-T-2F, PTB7-Th and PBDB-T-SF. The dashed lines in (c) are guides to the eye. For OSC device data and preparation conditions, we refer to the Supplementary Note 2. 
First, one intriguing observation is that the IQE averaged over the range of absorption of the BHJ, increases with the IE offset, as shown in Fig. 1(c). Second, IQEs are practically independent of donor or acceptor excitation (see IQE spectra in Supplementary Note 2). This implies that the same process governs the quantum efficiency, even though charge generation is expected to occur via electron transfer when exciting the donor, and hole transfer upon acceptor excitation, the former determined by the EA offset, the latter by the IE offset. In other words, the IE offset determines the IQE even when selectively exciting the donor, and despite large EA offsets in all systems. Third, the device fill factor, a measure of the field dependence of charge generation, increases with the IE offset as well, suggesting that in low offset systems, charge generation exhibits a stronger dependence on the external bias (see Supplementary Note 2).

To reveal the underlying reasons, we monitored the dynamics of excited states by transient absorption spectroscopy. Fig. 2 shows the transient absorption (TA) spectra of selected thin film blends after selective donor or acceptor photoexcitation. Further TA data can be found in Supplementary Note 3. Clearly, donor and acceptor photoexcitation generate initially different excited states: donor excitation leads to photobleaching of the donor's ground state absorption ( $\mathrm{PB}_{\mathrm{D}}$ in Fig. 2), as well as to photo-induced absorption (PA). Excitation of the acceptor, on the other hand, initially bleaches the ground state absorption of the acceptor only ( $\mathrm{PB}_{\mathrm{A}}$ in Fig. 2), associated with PA features resembling the excitonic signatures of neat acceptor films. While donor and acceptor photoexcitation initially generate different excited states (see red lines for the initial TA spectra in Fig. 2), the same TA spectra are observed after 300 ps (see blue lines in Fig. 2). We attribute this to charge generation, however not in the case of DR3:IEICO and PBDB-T-2F:IEICO, which we discuss below. To compare the excited state evolution after donor and acceptor excitation, we determined the ground state bleach integrated over the entire PB region (shaded areas in Fig. 2). Quantitatively, we observe that the relative photobleach area (indicating the overall excitation density) after charge generation (probed at $300 \mathrm{ps}$ ) compared to the initial photobleach area (probed at $0.4 \mathrm{ps}$ ), does not depend on donor or acceptor excitation, while it increases with the IE offset (Fig. 2(i)). 
a)
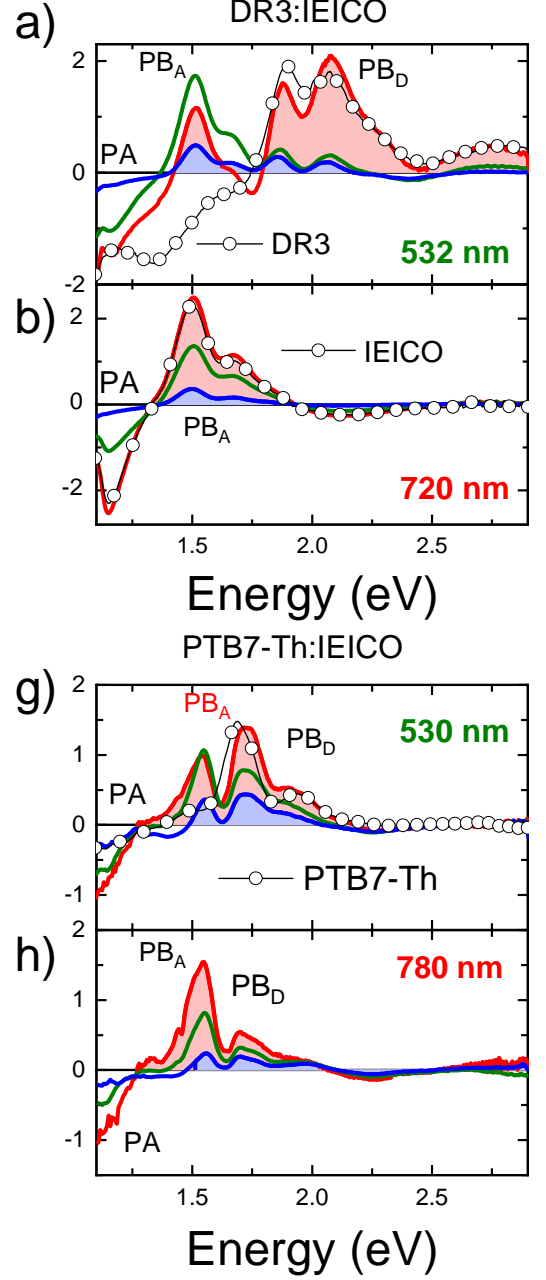

c)

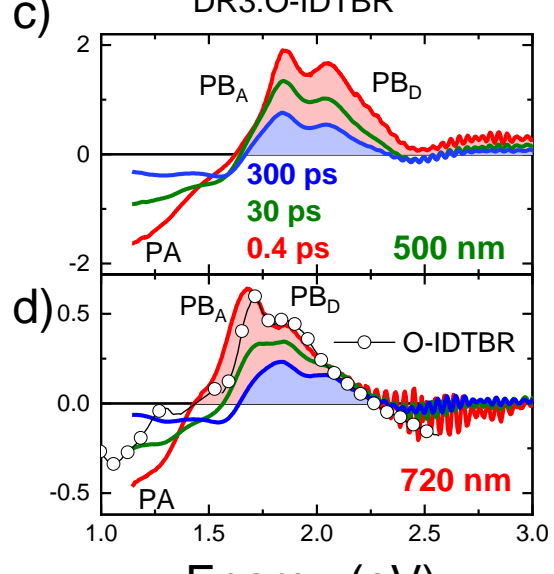

Energy $(\mathrm{eV})$

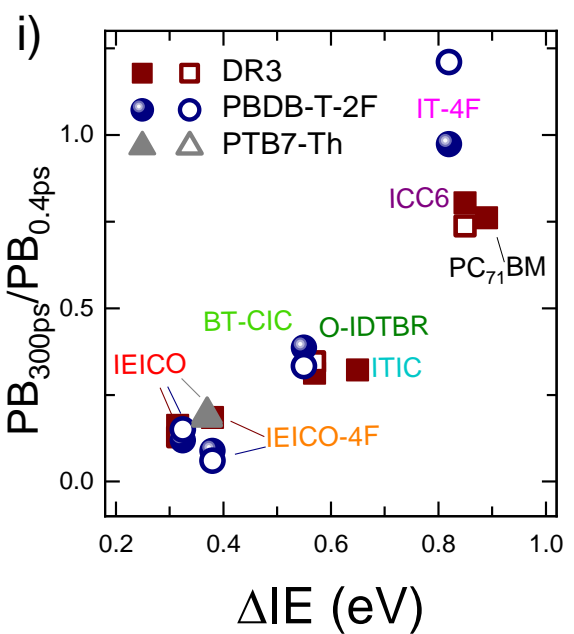

e)

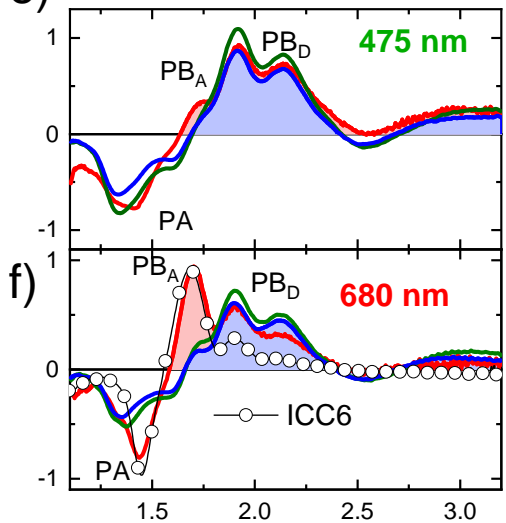

Energy $(\mathrm{eV})$

PBDB-T-2F:IT4F

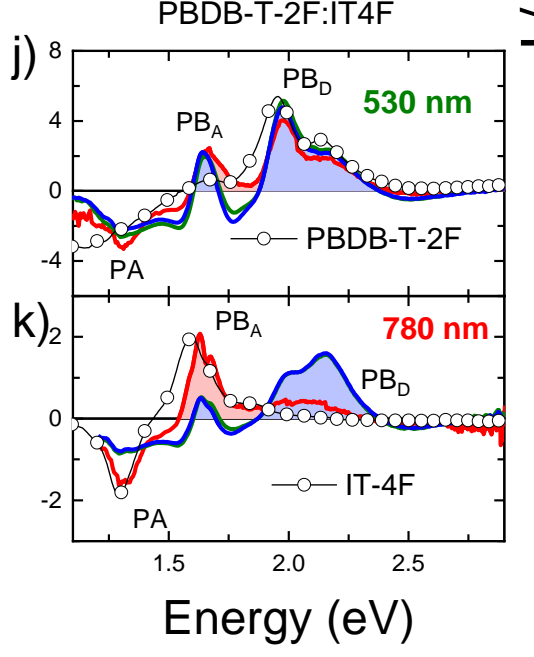

Fig. 2: The charge carrier generation yield is independent of donor or acceptor excitation, but it increases with the IE offset. (a-h) and (j-k) Picosecond-nanosecond transient absorption spectra of selected neat materials (open symbols) and blends (colored lines) after $0.4 \mathrm{ps}$ (red lines), 30 ps (green lines) and 300 ps (blue lines): (a-b) DR3:IEICO, (c-d) DR3:O-IDTBR, (e-f) DR3:ICC6, (g-h) PTB7-Th:IEICO, and (j-k) PBDB-T-2F:IT-4F blend films after selective excitation of donor (a, c, e, g, j) and acceptor molecules (b, d, f, $\mathrm{h}, \mathrm{k})$. (i) Ratio of the integrated photobleach (after $300 \mathrm{ps})$ to the initial photobleach $(0.4-0.7 \mathrm{ps})\left(\mathrm{PB}_{300 \mathrm{ps}} /\right.$ $\mathrm{PB}_{0.4 \mathrm{ps}}$ ) of all investigated blends for absorbed fluences of $\sim 1.6-4 \mu \mathrm{J} / \mathrm{cm}^{2}$, with donor (open symbols) and acceptor (closed symbols) excitation, as a function of the IE offset $(\Delta \mathrm{IE})$.

DR3:IEICO and PBDB-T-2F:IEICO blends are, however, very different. As shown in Fig. 2(a), within 30 ps after donor photoexcitation (see green line), the initially convoluted exciton-induced absorption signatures of donor and acceptor have evolved into the exciton-induced absorption of the acceptor only, while only marginal charge generation is observed. Besides, acceptor excitons created by direct photoexcitation of the acceptor subsequently decay to the ground state without undergoing charge transfer. We conclude that almost exclusively energy transfer (FRET) from the donor to the 
acceptor IEICO takes place, while hole transfer to the donor is inefficient. This is supported by steady-state PL quenching measurements, which show poor PL quenching upon acceptor excitation, and importantly, acceptor PL upon donor photoexcitation (Supplementary Note 4), supporting FRET occurs in these systems. We note that PL quenching efficiencies in the other blends are larger. This low exciton quenching efficiency in DR3:IEICO and PBDB-T-2F:IEICO blends cannot be attributed to morphological differences between the blends. In fact, we have evidence that all donor-acceptor blends are intimately mixed from transmission electron microscopy (TEM) combined with electron energy-loss spectroscopy (EELS) and scanning transmission electron microscopy (STEM) (Supplementary Note 5), showing no indication of component demixing that could satisfactorily explain the inefficient exciton quenching. Secondly, in more intermixed as-cast DR3:IEICO blends, obtained without solvent vapor annealing, even lower PL quenching efficiencies were observed upon acceptor excitation and enhanced energy transfer upon donor excitation (Supplementary Figure 24).

The large overlap of donor photoluminescence and acceptor absorption, shown in Fig. 3(a) and (b) actually triggers efficient energy transfer in all the donor:NFA systems. In fact, the FRET radii ${ }^{43,44}$ (Supplementary Note 6) are larger for the donor-NFA pairs than for the respective donor-donor pairs (see Fig. 3(c)). This implies that when approaching the donor-acceptor interface, an exciton in the donor is more likely to transfer to an acceptor than to reach the interface to undergo electron transfer. More precisely, for donor excitons at a distance of $1 \mathrm{~nm}$ from the interface, the calculated FRET rates are on the order of $10^{13} \mathrm{~s}^{-1}$ in all donor:NFA systems (Supplementary Note 6). This implies that FRET also competes with donor-acceptor electron transfer at shorter distances, as very recently also suggested by Karki et al. ${ }^{34}$

Indeed, FRET from DR3 to NFA could clearly be observed in solution (Supplementary Figure 15). In films, however, it is instantaneously followed by hole transfer from the acceptor to the donor. We confirmed ultrafast hole transfer in diluted DR3:NFA (95:5) blends upon selective excitation of the acceptor, which resulted in a rise of the donor signal within the response time of our TA setup of a few hundred femtoseconds (Supplementary Figure 16) in line with the recent report by Zhong et al. ${ }^{36}$ 

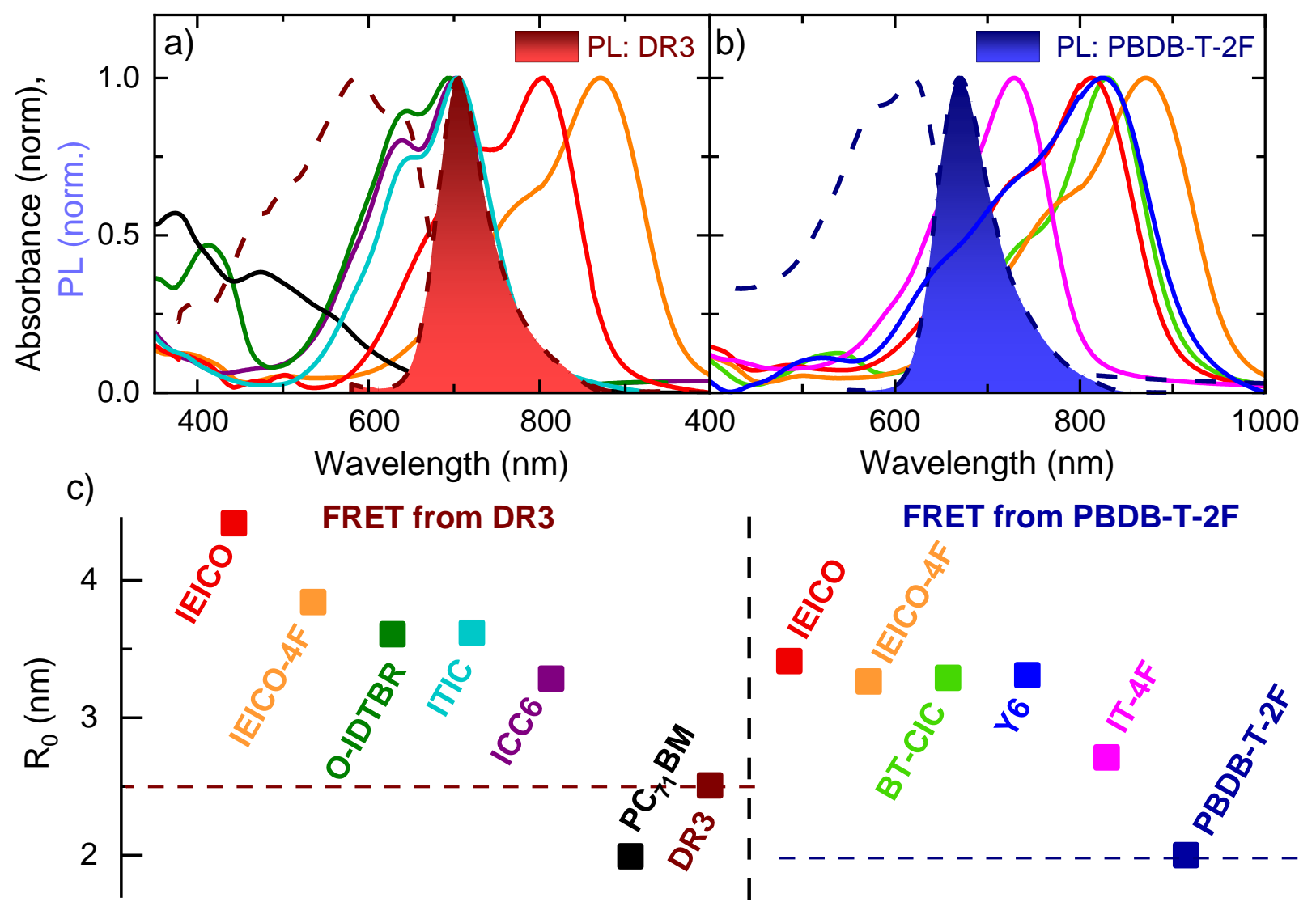

Fig. 3: Spectral overlap of donor photoluminescence and acceptor absorption facilitates ultrafast energy transfer. Normalized UV-Vis absorption spectra of (a) acceptors (continuous lines) used with DR3 as donor (dashed line) and (b) acceptors used with PBDB-T-2F as donor and photoluminescence spectra of DR3 and PBDB-T-2F (filled curves) as indicated by the legends. c) FRET radii for DR3- and PBDB-T-2F-based blends as well as within the donor phase.

As a result of FRET, the charge transfer in blends is largely hole transfer from the acceptor to the donor, independent of donor or acceptor photoexcitation. This explains why donor and acceptor photoexcitation yields similar IQEs. It also implies that IQE depends on the driving force of the hole transfer reaction, governed by the difference between the singlet excited state energy of the acceptor and the blend's CT state.

The conversion efficiency of the acceptor exciton to free charge carriers therefore controls the IQE. We identify the efficiency limit from the amount of acceptor exciton quenching. The exciton quenching efficiency was determined by time-resolved photoluminescence (TRPL) experiments (Fig. 4(a-d)). The efficiency of exciton quenching closely follows the IQE, implying that apart from the acceptor singlet exciton to CT state conversion, CT to free charge separation and carrier extraction are efficient. We emphasize that in NFA-based blends exciton quenching determined by TRPL experiments often provides an immediate measure of the free charge generation efficiency and thus 
device IQE, unlike in fullerene-based systems, in which ultrafast and efficient exciton quenching does not necessarily indicate high free carrier yields and IQE due to the occurrence of other loss channels such as geminate charge recombination. Finally yet importantly, we stress that the correlation of PL quenching from TRPL and IQE cannot be explained by donor-acceptor demixing, as morphology does not follow energetic offsets and a coincidental correlation across such a large set of different donor-acceptor blends appears highly unlikely (see also STEM and EELS maps in Supplementary Note 5).
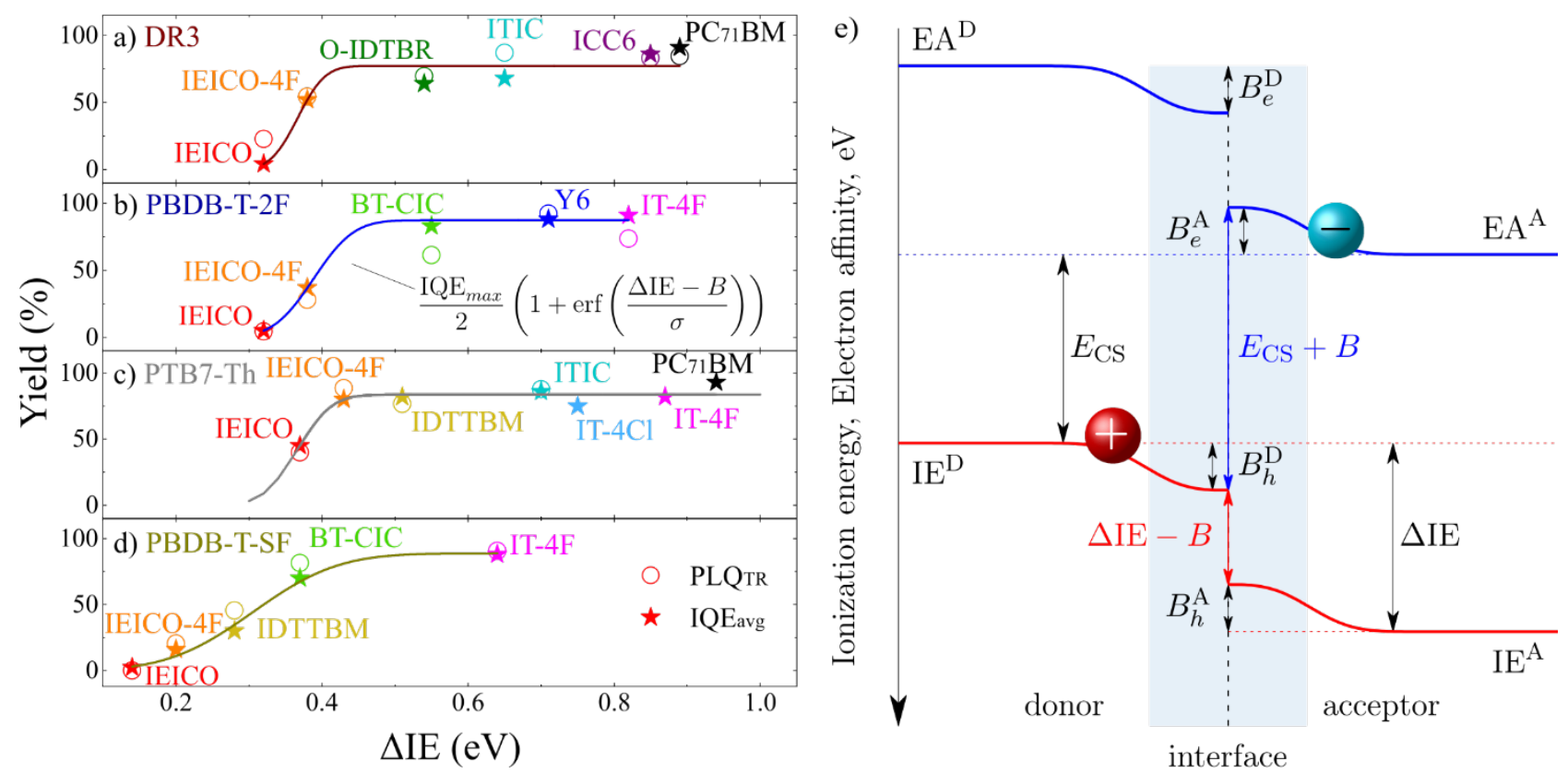

Fig. 4: The IQE follows the exciton quenching yield controlled by the IE offset and energy level bending at the D/A interface. Average internal quantum efficiency $\left(\mathrm{IQE}_{\mathrm{avg}}, \star\right)$ and exciton lifetime quenching 1$\tau_{\text {blend }} / \tau_{\text {pristine }}\left(\mathrm{PLQ}_{\mathrm{TR}}, \circ\right.$ ) as a function of ionization energy offset $(\Delta \mathrm{IE})$ for (a) DR3-, (b) PBDB-T-2F-, (c) PTB7-Th- and (d) PBDB-T-SF-based blends. The dependence corresponds to the fraction of the interface, which exhibits an energy bending lower than $\triangle \mathrm{IE}$, assuming a Gaussian distribution of average energy level bending $B$ and a standard deviation $\sigma$ of $0.37 \mathrm{eV}$ and $0.04 \mathrm{eV}$ for DR3, $0.37 \mathrm{eV}$ and $0.05 \mathrm{eV}$ for PTB7-Th, $0.39 \mathrm{eV}$ and $0.06 \mathrm{eV}$ for PBDB-T-2F and $0.31 \mathrm{eV}$ and $0.13 \mathrm{eV}$ for PBDB-T-SF. (e) Schematic of the energy level bending at the donor-acceptor heterojunction due to the electrostatic interaction of charges with quadrupole moments of surrounding molecules.

Since the transition from the acceptor singlet exciton to the interfacial charge transfer state is a hole transfer from the acceptor to the donor, it comes as little surprise that it depends on the IE offset. It is, however, surprising and unclear why a system-independent offset as large as $0.3-0.4 \mathrm{eV}$ is required to obtain a $50 \%$ charge transfer yield and why the efficiency increase is less steep than that of a typical thermally-activated process. 
To explain both observations, we show an energy level diagram of a solar cell in Fig. 4(e). Here, the key difference to common diagrams is the bending of the ionization energy (IE) and electron affinity (EA) at the interface. This energy level bending is due to the gradual change in the electrostatic potential created by the quadrupole moments of the NFA and the donor polymer. ${ }^{45-51}$ The energy level is bent in a direction that corresponds to the (negative) sign of the in-plane quadrupole moment of the acceptor-donor-acceptor (A-D-A) NFA molecule. Because of energy level bending, the CT state energy increases, and the IE difference narrows by approximately the same amount at the interface. The energy level bending has no impact on the diffusion of excitons towards the interface, as both EA and IE in a material are bent in the same direction, leaving the energy gap within the acceptor (or donor) unchanged.

Energy level bending has a clear impact on the energetics of two-particle interactions of states involved in charge generation and separation: the excited state of the acceptor $\left(\mathrm{A}^{*}\right)$, the charge transfer state $(\mathrm{CT})$, and the charge separated state $(\mathrm{CS})$. For the acceptor exciton, $E^{\mathrm{A}^{*}}=\mathrm{EA}^{\mathrm{A}}-\mathrm{IE}^{\mathrm{A}}-$ $E_{\text {Coulomb }}^{\mathrm{A}^{*}}$, where $E_{\text {Coulomb }}^{\mathrm{A}^{*}}$ is the Coulomb interaction energy of the electron and hole, in the solid state. Different signs of energy level bending on the donor and acceptor sides increase the energy of the charge transfer state to $E^{\mathrm{CT}}=\mathrm{EA}_{\text {interface }}^{\mathrm{A}}-\mathrm{IE}_{\text {interface }}^{\mathrm{D}}-E_{\text {Coulomb }}^{\mathrm{CT}}=\mathrm{EA}^{\mathrm{A}}-\mathrm{IE}^{\mathrm{D}}+B-$ $E_{\text {Coulomb }}^{\mathrm{CT}}$. Here $E_{\text {Coulomb }}^{\mathrm{CT}}$ is the Coulomb binding of a CT state at a perfectly flat interface (i.e., without bending), and $B=B_{e}^{\mathrm{A}}+B_{h}^{\mathrm{D}}$ is the bias potential, as depicted in Fig. 4(e). Finally, the CS state is not affected by the energy level bending, since both electron and hole are away from the interface, $E^{\mathrm{CS}}=\mathrm{EA}^{\mathrm{A}}-\mathrm{IE}^{\mathrm{D}}$.

Now we look at the dissociation energy of the CT state, i.e., the CT to CS transition, $\Delta E_{\mathrm{CT} \rightarrow \mathrm{CS}}=$ $E^{\mathrm{CS}}-E^{\mathrm{CT}}=E_{\mathrm{Coulomb}}^{\mathrm{CT}}-B$. Clearly, the interfacial bias reduces the dissociation energy of the charge transfer state. In fact, the optimal condition is achieved for $B=E_{\mathrm{Coulomb}}^{\mathrm{CT}} \sim 0.4 \mathrm{eV}$, which then provides barrier-less dissociation, as demonstrated very recently for Y $6 .{ }^{29}$ Interestingly, most of the reported blends provide sufficient bias to split the charge transfer state, which explains why they are quite efficient.

We now turn to the excited to charge transfer state transition. The driving force for this reaction, $\Delta E_{\mathrm{A}^{*} \rightarrow \mathrm{CT}}=E^{\mathrm{A}^{*}}-E^{\mathrm{CT}}=\Delta \mathrm{IE}-B-\left(E_{\text {Coulomb }}^{\mathrm{A} *}-E_{\mathrm{Coulomb}}^{\mathrm{CT}}\right)$, has three contributions. First, the offset between the ionization energies of the donor and the acceptor, $\Delta \mathrm{IE}=\mathrm{IE}^{\mathrm{D}}-\mathrm{IE}^{\mathrm{A}}$. Second, the negative contribution of the bias potential, since the CT state energy is increased by the energy level bending (see Fig. 4 (e)). Third, the difference between the hole-electron binding energies of a Frenkel-type exciton and a charge transfer state. It might seem that the last term provides a large negative contribution to the driving force, since it takes a substantial amount of energy to pull an 
electron and hole apart from each other during an excited to charge transfer state transition, in a molecular dimer. However, polarizable force field calculations demonstrate that, in a solid state, strong dielectric stabilization of two charges in a CT state compensates Coulomb binding of a Frenkel exciton, i.e., $E_{\text {Coulomb }}^{A *}-E_{\text {Coulomb }}^{C T} \sim 0$. As a result, spontaneous dissociation of an excited state has recently been observed experimentally, even in neat NFA films, that is, in the absence of an electron donor (hole acceptor). ${ }^{52}$

The consequence is that $\Delta E_{\mathrm{A}^{*} \rightarrow \mathrm{CT}}$ is reduced to $\Delta \mathrm{IE}-B$, which can be either positive or negative, depending on $\triangle \mathrm{IE}$. This is experimentally confirmed by electroluminescence (EL) spectroscopy of NFA-based solar cells: here, BHJs with large $\triangle \mathrm{IE}$, namely DR3:PCBM, DR3:ICC6, DR3:ITIC, PBDB-T-2F:IT-4F, exhibit a clear CT state EL emission observed at lower energy than the neat acceptor's EL, while no such CT state emission is observed in BHJs with low offsets (see Supplementary Note 7). We hypothesize that excitons reaching an interface that exhibits a negative $\Delta E_{\mathrm{A}^{*} \rightarrow \mathrm{CT}}$ have the chance to diffuse back to the bulk of the acceptor phase. Hence, the exciton lifetime as measured by TRPL gradually becomes similar to that of a neat acceptor film when $\Delta \mathrm{IE}$ decreases (Fig. 4 (a), (b), (c)).

To further understand the gradual increase of the IQE with the IE offset $(\Delta \mathrm{IE})$, we note that rough interfaces provide a broad spread of available interfacial biases. Assuming a Gaussian distribution of $B$ and integrating the fraction of excited to $\mathrm{CT}$ state transitions for which $\Delta \mathrm{IE}-B$ is positive, we obtain $\mathrm{IQE}=\frac{\mathrm{IQE}_{\max }}{2} \operatorname{erfc} \frac{B-\Delta \mathrm{IE}}{\sigma}$, where $B$ is now the mean value of the bias potential. The fit to this simple expression describes the experimentally observed IQE vs. $\triangle$ IE dependence very well across all investigated systems, as shown in Fig. 4 (a-d). Remarkably, the fits provide similar average energy level bending $B$ for DR3, PCE10, and PM6 as donors, precisely $B \sim 380 \pm 10 \mathrm{meV}$, standard deviation of the bending distribution $\sigma \sim 50 \pm 10 \mathrm{meV}$ and $\mathrm{IQE}_{\max } \sim 80 \pm 4 \%$. We note that for the PBDB-T-SF:NFA systems a smaller value of $B \sim 310 \mathrm{meV}$ with a broader distribution $(\sigma=130$ $\mathrm{meV}$ ) is observed. However, as for the other three donors, the same minimum ionization energy offset of $0.5 \mathrm{eV}$ is required to efficiently split $\mathrm{CT}$ states, i.e., to compensate the energy level bending of $0.5 \mathrm{eV}$. Within our model, the broader distribution of the bias potential $B$ implies an (electrostatically) less ordered donor-acceptor interface.

The DR3-based systems exhibit a small additional increase in IQE with increasing IE offset, beyond the steep initial rise. This is most likely caused by incomplete charge separation, as indicated by the IE-offset dependent fraction of geminate recombination (Supplementary Note 3.4) possibly due to a larger CT state binding energy with DR3 than with conjugated donor polymers, related to a smaller hole delocalization. 
To summarize, the interfacial bias potential and associated interfacial energy level bending plays a dual role: it facilitates the dissociation of the charge transfer state, but at the same time it reduces the driving force for the exciton to CT state conversion. For a barrierless CT state dissociation biases of at least $0.4 \mathrm{eV}$, that is the $\mathrm{CT}$ state Coulomb binding energy, are required.

\section{Conclusion}

We demonstrated that ultrafast energy transfer from either small molecule or polymer donors to lowbandgap acceptors in NFA-based solar cells competes with donor to acceptor electron transfer. Energy transfer funnels excitons from donor to acceptor, renders the electron affinity offset virtually unimportant, and leads to a charge generation efficiency exclusively dependent on the ionization energy offset. Furthermore, for small ionization energy offsets, an energy barrier for the acceptor exciton to charge transfer state transition exists. This barrier is due to the interfacial energy level bending by the electrostatic fields created by the NFA molecular quadrupole moments. As a result, IE offsets larger than the charge transfer state Coulomb binding energy are required for barrierless hole transfer from the acceptor to the donor. In fact, IE offsets of $0.5 \mathrm{eV}$ are needed to ensure closeto-unity charge generation efficiency. This sets an intrinsic limit to the minimum energy losses that can be tolerated in NFA-based systems without sacrificing the charge separation efficiency, in other words, ensuring close-to-unity quantum efficiency. It also pinpoints additional system parameters that can be used to further improve the device efficiency. Specifically, the largest fraction of losses that limit the $\mathrm{V}_{\mathrm{OC}}$ is related to the electron-hole Coulomb binding of a CT state and the disorder of the interfacial electrostatic bias potential. Since Coulomb binding of the CT state in NFAs is of the order of $0.4 \mathrm{eV}$, and close-to unity charge generation efficiency is achieved for $0.5 \mathrm{eV}$, we can, in principle, further reduce energetic losses by ca. $0.1 \mathrm{eV}$, when decreasing the disorder of the electrostatic bias. Further improvements can be achieved by reducing non-geminate (non-radiative) recombination of charges and charge transfer states. Additional improvements will require conceptually new designs that either decrease the Coulomb binding energy of the CT state or generate charges without passing through a CT state intermediate, e.g., by tunneling directly from the excited to the CS state. Apart from the energy losses, the pragmatic way to improve overall OSC efficiency is to increase the absorption coefficient of the donor and acceptor and therefore push the EQE towards unity, by avoiding carrier recombination losses typical for thicker photoactive layers. 


\section{Methods}

\section{Materials}

PBDB-T-2F, IT-4F, Y6, IEICO-4F were purchased from Solarmer Materials Inc. DR3TBDTT (DR3), PTB7-Th, ITIC, ICC6 (IDIC), BT-CIC, IT-4Cl, IEICO and Phen-NaDPO were purchased from 1-Material Inc. IDTTBM ${ }^{53}$ and O-IDTBR ${ }^{54}$ were synthesized at KAUST. PC 71 BM was obtained from Solenne BV, PEDOT:PSS from Heraeus and PBDB-T-SF and PFN-Br from Organtec Ltd. Chlorobenzene anhydrous $99.8 \%$, carbon disulfide anhydrous $\geq 99 \%$ and 1,8 -diiiodooctane $98 \%$ were purchased from Sigma-Aldrich, chloroform from VWR, 1-chloronaphtalene from Fluka and dimethydisulfide 99\% from Alfa Aesar. All materials were used as received.

Full material names: IT-4Cl: 3,9-bis(2-methylene-((3-(1,1-dicyanomethylene)-chloro)-indanone))5,5,11,11-tetrakis(4-hexylphenyl)-dithieno[2,3-d:2',3'-d']-s-indaceno[1,2-b:5,6-b']dithiophene, IT4F: 3,9-bis(2-methylene-((3-(1,1-dicyanomethylene)-6,7-difluoro)-indanone))-5,5,11,11-tetrakis(4hexylphenyl)-dithieno[2,3-d:2',3'-d']-s-indaceno[1,2-b:5,6-b']dithiophene, ITIC: 3,9-bis(2methylene-(3-(1,1-dicyanomethylene)-indanone)-5,5,11,11-tetrakis(4-hexylphenyl)-dithieno[2,3d:2',3'-d']-s-indaceno[1,2-b:5,6-b']-dithiophene, Y6: (2,20 -((2Z,20 Z)-((12,13-bis(2- ethylhexyl)3,9-diundecyl-12,13-dihydro-[1,2,5] thiadiazolo[3,4-e] thieno[2,"30 ' :4',50 ] thieno[20 ,30 :4,5]pyrrolo[3,2-g] thieno[20,30 :4,5] thieno[3,2-b]indole-2,10-diyl)bis(methanylylidene))bis(5,6difluoro-3-oxo-2,3-dihydro-1H-indene-2,1-diylidene))dimalononitrile). ICC6: indacenodithiophene end capped with 1,1-dicyanomethylene-3-indanone. IEICO-4F: 2,2'-[[4,4,9,9-tetrakis(4hexylphenyl)-4,9-dihydro-s-indaceno[1,2-b:5,6-b']dithiophene-2,7-diyl]bis[[4-[(2-ethylhexyl)oxy]5,2-thiophenediyl]methylidyne(5,6-difluoro-3-oxo-1H-indene-2,1(3H)-

diylidene)]]bis[propanedinitrile], O-IDTBR： 5,5'-[(4,9-dihydro-4,4,9,9-tetraoctyl-s-indaceno[1,2b:5,6-b']dithiophene-2,7-diyl)bis(2,1,3-benzothiadiazole-7,4-diylmethylidyne)]bis[3-ethyl-2thioxo-4-Thiazolidinone, BT-CIC: 4,4,10,10-tetrakis(4-hexylphenyl)-5,11-(2-ethylhexyloxy)-4,10dihydrodithienyl[1,2-b:4,5b']benzodithiophene-2,8-diyl)bis(2-(3-oxo-2,3-dihydroinden-5,6dichloro-1-ylidene)malononitrile), IEICO: 2,2'-((2Z,2'Z)-((5,5'-(4,4,9,9-tetrakis(4-hexylphenyl)4,9-dihydro-s-indaceno[1,2-b:5,6-b']dithiophene-2,7-diyl)bis(4-((2-ethylhexyl)-oxy)thiophene-5,2diyl))bis(methanylylidene))bis(3-oxo-2,3-dihydro-1H-indene-2,1-diylidene))dimalononitrile,

IDTTBM: indacenodithienothiophene 2-(benzo[c][1,2,5]thiadiazol-4-ylmethylene)malononitrile, PC 71 BM: [6,6]-Phenyl-C71-butyric acid methyl ester. Phen-NaDPO: ((2-(1,10-phenanthrolin-3yl)naphth-6-yl)diphenylphosphine oxide), PEDOT:PSS: $\operatorname{poly}(3,4-$ ethylenedioxythiophene):poly(styrenesulfonate), PFN-Br: $\quad \operatorname{Poly}(9,9-b i s(3$ '-(N,N-dimethyl)-Nethylammoinium-propyl-2,7-fluorene)-alt-2,7-(9,9-dioctylfluorene))dibromide. PBDB-T-2F: 
Poly[(2,6-(4,8-bis(5-(2-ethylhexyl-3-fluoro)thiophen-2-yl)-benzo[1,2-b:4,5-b']dithiophene))-alt(5,5-(1',3'-di-2-thienyl-5',7'-bis(2-ethylhexyl)benzo[1',2'-c:4',5'-c']dithiophene-4,8-dione)], PBDB-T-SF: $\quad$ Poly[(2,6-(4,8-bis(5-(2-ethylhexylthio)-4-fluorothiophen-2-yl)-benzo[1,2-b:4,5b']dithiophene))-alt-(5,5-(1',3'-di-2-thienyl-5',7'-bis(2-ethylhexyl)benzo[1',2'-c:4',5'-c']dithiophene4,8-dione)], PTB7-Th: Poly([2,6'-4,8-di(5-ethylhexylthienyl)benzo[1,2-b;3,3-b]dithiophene]\{3fluoro-2[(2-ethylhexyl)carbonyl]thieno[3,4-b]thiophenediyl $\})$, DR3: (5Z,5'E)-5,5'-((4,8-bis(5-(2ethylhexyl)thiophen-2-yl)benzo[1,2-b:4,5-b']dithiophene-2,6-diyl)bis(3,3"-dioctyl-[2,2':5',2"terthiophene]-5",5-diyl))bis(methaneylylidene))bis(3-ethyl-2-thioxothiazolidin-4-one).

\section{Thin-film and device preparation}

Organic photovoltaic (OPV) cells were fabricated using indium-tin-oxide (ITO)-coated glass substrate with a sheet resistance of $10 \Omega$.sq. ${ }^{-1}$. Substrates were cleaned by sequential ultrasonication in dilute Extran 300 detergent solution, deionized water, acetone, and isopropyl alcohol for $10 \mathrm{~min}$ each. The substrates were then subjected to a UV-ozone treatment for $10 \mathrm{~min}$. For normal architecture solar cells, a thin layer $(\approx 30 \mathrm{~nm})$ of PEDOT:PSS was spin-coated onto the UV-treated substrates and then dried on a heating plate at $150{ }^{\circ} \mathrm{C}$ for $10 \mathrm{~min}$. For inverted solar cells, zinc oxide $(\mathrm{ZnO})$ sol-gel was spin-coated on cleaned glass/ITO substrates with a spin speed of $4000 \mathrm{rpm}$ for 30 seconds, yielding a thickness of ca. $20 \mathrm{~nm}$. The as-cast films were then thermally annealed in air at $150{ }^{\circ} \mathrm{C}$ for $10 \mathrm{~min}$ and allowed to cool down to room temperature. Active layers were obtained by dissolving donor and acceptor in organic solvents (chloroform or chlorobenzene) inside the glovebox. The asprepared solutions were stirred for at least $1 \mathrm{~h}$ at $40-50{ }^{\circ} \mathrm{C}$ before being cast on the substrates. The active layers were spin-cast from the solutions at $50{ }^{\circ} \mathrm{C}$ for $45 \mathrm{~s}$, using a programmable spin-coater from Specialty Coating Systems (Model G3P-8). The active layers were then annealed (solvent vapor annealed (SVA) for all-small molecule cells and thermally annealed for polymer-based cells). For normal architecture cells, a layer of $5 \mathrm{~nm}$ of Phen-NaDPO (or PFN-Br for polymer-based cells) as the electron-transport layer (ETL) was spun from methanol solution $\left(0.5 \mathrm{mg} \cdot \mathrm{mL}^{-1}\right)$ on top of the BHJ layer. Next, the samples were placed in a thermal evaporator for evaporation of a $100 \mathrm{~nm}$-thick layer of aluminum evaporated at $5 \AA . \mathrm{s}^{-1}$ at a pressure of less than $2 \times 10^{-6}$ Torr. For inverted architecture devices, $7 \mathrm{~nm}$ molybdenum(VI) oxide $\mathrm{MoO}_{3}$ (Alfa) and $100 \mathrm{~nm} \mathrm{Ag} \mathrm{(Kurt} \mathrm{Lesker)} \mathrm{were} \mathrm{evaporated.}$ The effective area of the tested solar cells is $0.1 \mathrm{~cm}^{2}$. This effective area was determined from the layout of ITO substrate and top contact mask. Details of active-layer preparation and optimization are given in Supplementary Note 2. 


\section{Device characterization}

$J-V$ characteristics of solar cells were measured in a nitrogen-filled glove box. Solar cells were illuminated with light from an Oriel Sol3A Class AAA solar simulator calibrated to 1 sun, AM1.5G, with a KG-5 silicon reference cell certified by Newport. A Keithley 2400 source meter was used to measure the $J-V$ curves in the reverse direction, that is, from positive to negative bias with a dwell time of $10 \mathrm{~ms}$. For each $J-V$ curve 100 data points were collected. The solar cells' external quantum efficiency (EQE) was measured with a commercial setup (PV Measurement Inc.) at zero bias. Solar cells were illuminated with light supplied from a Xenon arc lamp in combination with a dual-grating monochromator. For each wavelength, the number of incident photons was calculated using a calibrated silicon photodiode. Calibration was performed by The National Institute of Standards and Technology (NIST). The internal quantum efficiency (IQE) of OPV devices was calculated using: $\operatorname{IQE}(\%)=\operatorname{EQE}(\%) /(100 \%$ - Reflectance $(\%)$ - Parasitic Absorption (\%)). The reflectance spectra were measured in an integrating sphere using the same EQE setup, while the parasitic absorption spectra were obtained by transfer matrix modelling of the device stack.

\section{Ultraviolet photoelectron (UPS) and low-energy inverse photoelectron (IPES) spectroscopy.}

The substrate for film deposition was $\mathrm{Au}(100 \mathrm{~nm}) / \mathrm{Cr}(10 \mathrm{~nm}) / \mathrm{Si}$ (n-type), with the $\mathrm{Au}$ and $\mathrm{Cr}$ evaporated and sputtered within an Angstrom evaporation/sputter tool, respectively. Solutions of 2$5 \mathrm{mg} \cdot \mathrm{ml}^{-1}$ in chloroform were prepared and stirred overnight in a glove box and then spin-coated (5000 rpm for 1 minute) onto an Ar+ ion sputtered Au substrate and then transferred to UHV for analysis. The organic small molecule/polymer film thickness was 10-20 nm.

UPS was performed with a vacuum ultraviolet (VUV) He (1) discharge line $21.22 \mathrm{eV}$ (focus) and a Sphera II EAC 125 7-channeltron electron analyzer. A small component ca. $2 \% \beta$ (23.09 eV) was present. The sample was positioned at $0^{\circ}$ with respect to the analyzer-to-sample plane. Fermi level calibration was performed using an Ar+ sputtered clean metallic silver (Ag) foil in electrical contact to the manipulator holding the sample in contact with the electron analyzer. During UPS a bias of $9.97 \mathrm{eV}$ was applied to observe the secondary electron cut-off. Subsequent measurements were made in electrical contact with thin films to prevent surface charging. UPS Scans were conducted from low to high kinetic energy at a constant analyzer pass energy of $10 \mathrm{eV}$ and subsequent scans were carefully monitored to detect beam-induced degradation or evidence of charging.

An in-house built UHV LE-IPES set-up was used following closely a design outlined by Yoshida ${ }^{41}$ which was also developed from a prior design. This operates in the Bremsstrahlung Isochromatic Mode (BIS). In brief, an incident electron beam (dispersion of $0.25-0.5 \mathrm{eV}$ ) is directed at $0^{\circ}$ with respect to the sample plane, and the electron energy is swept slowly. The outgoing light from the 
surface was collected by placing an external focusing lens and an internal vacuum collimating lens with a detector and all further stray light shielded. This was conducted with a low energy electron source (Staib) operating in the range of 20-30 eV energy ( $\mathrm{BaO}$ cathode Heatwaves), with a retarding bias of $+20 \mathrm{~V}$ applied to the sample in good electrical contact. As stated, the photons were collected outside of vacuum by a solid-state PMT detector (Hamamatsu R585) (0.5-1h, integration time 1020s, 200 data points), facing the sample within a narrow wavelength window using a bandpass filter of $280 \mathrm{~nm}$ (Semrock) (10 nm width). This measurement was performed consecutively without either exposure to $\mathrm{Al} \mathrm{K \alpha} \mathrm{X}$-ray $1486.6 \mathrm{eV}$ for XPS analysis or re-exposure of the surface to ambient atmosphere in a base pressure of $10^{-9}$ mbar to accurately join with UPS spectra. More information can be found in the Supplementary Note 1.

\section{Transmission electron microscopy (TEM)}

TEM studies in combination with EELS were performed on a Thermo Fischer (former FEI) Titan 80-300 TEM equipped with an electron monochromator and a Gatan Imaging Filter (GIF) Quantum 966. Details can be found in the Supplementary Information.

\section{Transient absorption spectroscopy (TA)}

Transient absorption (TA) pump-probe measurements were carried out with a home-built setup. For femto- to nanosecond pump-probe experiments, the following setup was used:

The output of a titanium:sapphire amplifier (Coherent LEGEND DUO, $4.5 \mathrm{~mJ}, 3 \mathrm{kHz}, 100 \mathrm{fs}$ ) was split and used to pump two optical parametric amplifiers (OPA) (Light Conversion TOPAS Prime), generating a tunable femtosecond pump beam and a signal wavelength at $1300 \mathrm{~nm}$. A white light supercontinuum was generated in a calcium fluoride / sapphire crystal pumped at $1300 \mathrm{~nm}$ (or @800 $\mathrm{nm}$ from the Ti:Sa amplifier). Pump and probe beams were delayed with respect to each other by a mechanical delay stage (Newport M-IMS600CCHA), guided to the sample by reflective optics and then focused and overlapped on the sample. During the measurements, the samples were kept under a dynamic vacuum of $<10^{-5}$ mbar. The transmitted part of the supercontinuum was dispersed in a custom-made prism spectrograph (Entwicklungsbüro Stresing) and detected by a 512-pixel NMOS linear image sensor (HAMAMATSU S8381-512). For the $1 \mathrm{~ns}$ to $300 \mu$ s delay (long delay) TA measurement, the same probe white-light supercontinuum was used as for the 100 fs to 8 ns delays. Here the excitation light (pump pulse) was provided by an actively Q-switched Nd:YVO4 laser (InnoLas picolo AOT) frequency-doubled to provide pulses at $532 \mathrm{~nm}$. Further details can be found in the Supplementary Information. 


\section{Time-resolved photoluminescence spectroscopy (TRPL)}

TRPL experiments were performed with a Streak Camera system (Hamamatsu C10910) with a temporal resolution of 1.4 ps. Samples were excited with the wavelength-tunable output of an OPO (Radiantis Inspire HF-100), itself pumped by the fundamental of a Ti:Sa fs-oscillator (Spectra Physics MaiTai eHP) and a Modelocked Ti:Sa (Chameleon Ultra I from Coherent) at $820 \mathrm{~nm}$. The PL of the samples was collected by an optical telescope (consisting of two plano-convex lenses) and focused onto the slit of a spectrograph (PI Spectra Pro SP2300) attached to the Streak Camera. Further details can be found in the Supplementary Information.

\section{Sensitive EQE and electroluminescence (EL) spectroscopy}

The high-dynamic range EQE spectra were collected at short-circuit conditions using focused monochromatic illumination from a Xenon arc lamp combined with a monochromator. The light was modulated by an optical chopper at $275 \mathrm{~Hz}$. The device output current was measured as a function of the incident photon energy using a lock-in amplifier (Stanford Instruments SR 830). The lamp intensity was calibrated with $\mathrm{Ge}$ and $\mathrm{Si}$ photo-diodes.

Electroluminescence (EL) measurements were carried out on devices (active area of $0.1 \mathrm{~cm}^{2}$ ) biased with DC voltages (Keithley 2420) similar to or lower than $V_{O C}$ at 1 sun illumination. The emission was collected by a collimator inside a nitrogen-filled glovebox and guided to a spectrograph (Princeton Instruments SP-2300) by an optical fiber. The spectrometer was equipped with a cooled Si CCD (Princeton Instruments PIX100BRX) and cooled InGaAs (Princeton Instruments PYR1024) camera. The system was wavelength calibrated with a Ne/Ar calibration light source (Princeton Instruments IntelliCal). The visible and infrared spectra were assembled at the $933 \mathrm{~nm}$ where they share the same relative sensitivity.

\section{Computer simulations}

Solid state ionization energies were evaluated in a perturbative way, starting from the gas-phase quantum chemical calculations (B3LYP/6-311g(d,p) level as implemented in the GAUSSIAN 09 package ${ }^{55}$ ) and then taking into account solid-state contribution as a perturbation, where electrostatic and induction energies are the first- and second-order corrections. These contributions to site energies were calculated self-consistently using the Thole model ${ }^{56,57}$ using atomic polarizabilities and distributed multipoles using GDMA program ${ }^{58}$ for a cation and a neutral molecule. This approach, in combination with an aperiodic inclusion of charges to a neutral periodic morphology, is available in the VOTCA-CTP package. Further details are given in Supplementary Information. 


\section{Author contributions}

S.K. performed the transient spectroscopy experiments, data analysis, and prepared the first draft of the manuscript. J.G. conceived the study, analyzed the transient and steady-state spectroscopic experiments, carried out the FRET calculations, and contributed to the narrative of the manuscript. Y.F. performed the DR3, PCE10, and PM6-based solar cell device preparation and characterization, and did the ellipsometry measurements and analysis. N.C. prepared and characterized PCE13-based devices and prepared thin film blends for TRPL experiments. C.S.D.C. prepared samples and characterized the steady-state absorption of thin films, PLQY, and PL quenching in DR3, PCE10, and PM6-based blends via TRPL. G.H. performed the UPS and LE-IPES experiments and data analysis. J.I.K. provided steady-state absorption, PL, and TRPL data of PCE13-based blends. A.M. calculated the solid-state IEs, EAs, and bias potentials. W.L. performed quantum-chemical calculations of donor-acceptor dimers. A.H.B. and T.A.D.P. determined the CT state energies from EL spectra. R.-Z.L. prepared DR3-based devices and characterized their performance. W.Z. synthesized the O-IDTBR acceptor. Y.L. prepared thin films for steady-state characterization. Da.A. performed thin film imaging and EELS analysis. E.A. contributed to the development and maintenance of the ultrafast laser spectroscopy setups. S.H.K.P determined the IQE of PCE10-based devices. A.S. determined the IE and EA of IEICO-4F and IT-4F by UPS and LE-IPES, respectively. P.M.B. supervised the device preparation of DR3-based solar cells and synthesis of IDTTBM used in this work. S.D.W. oversaw the UPS/LE-IPES experiments and data analysis. I.M. supervised the synthesis of OIDTBR used in this work. T.A. supervised the PBDB-T-2F donor device preparation and characterization. D.B. supervised the PTB7-Th donor device preparation and characterization. De.A. supervised the computational part of the work, conceived the model describing the energy level bending, and contributed to the narrative of the manuscript. F.L. conceived the study and supervised the steady-state and transient optical spectroscopy experiments and data analysis as well as the PBDB-T-SF donor device preparation and characterization and contributed to the narrative of the manuscript. All authors contributed to the revision of the final version of the manuscript.

\section{Competing interests}

The authors declare no competing interest.

\section{Data availability}

The datasets generated and analysed during this study and presented in this Article are available online at 'figshare link (to be provided)'. 


\section{Code availability}

The codes or algorithms used to analyze the data reported in this study are available from the corresponding authors upon reasonable request.

\section{Acknowledgements}

This publication is based upon work supported by the King Abdullah University of Science and Technology (KAUST) Office of Sponsored Research (OSR) under Award No: OSR-2018CARF/CCF-3079 and Award No. OSR-CRG2018-3746. De. A. acknowledges funding from the BMBF grant InterPhase and MESOMERIE (FKZ 13N13661, FKZ 13N13656) and the European Union Horizon 2020 research and innovation program ' Widening materials models' under Grant Agreement No. 646259 (MOSTOPHOS). De. A. also acknowledges the KAUST PSE Division for hosting his sabbatical in the framework of the Division's Visiting Faculty program. A.M. acknowledges postdoctoral support of the Alexander von Humboldt Foundation. The authors would like to thank Dr. Lutfan Sinatra of KAUST and Quantum Solutions LLC for assisting with the PLQY measurements. G.T.H would like to acknowledge Dr. Kenneth Graham and Dr. Aram Amassian (and previous group members including Dr. Max Tietze and Dr. Guy Olivier Ngongang Ndjawa) for having designed and installed and worked on the IPES setup. In particular for Dr. Kenneth Grahams assistance during trouble shooting during system optimization, as well as Mr. Usman Sharif for technical assistance.

\section{References}

1 Yang, C. et al. Effects of energy-level offset between a donor and acceptor on the photovoltaic performance of non-fullerene organic solar cells. Journal of Materials Chemistry A, doi:10.1039/C9TA04789A (2019).

2 Hyojung, $C$. et al. Influence of Blend Morphology and Energetics on Charge Separation and Recombination Dynamics in Organic Solar Cells Incorporating a Nonfullerene Acceptor. Advanced Functional M aterials 28, 1704389, doi:doi:10.1002/adfm.201704389 (2018).

3 Kaake, L. G. Towards the Organic Double Heterojunction Solar Cell. The Chemical Record 19, 11311141, doi:10.1002/tcr.201800180 (2019).

4 Menke, S. M., Ran, N. A., Bazan, G. C. \& Friend, R. H. Understanding Energy Loss in Organic Solar Cells: Toward a New Efficiency Regime. Joule 2 25-35, doi:https://doi.org/10.1016/j.joule.2017.09.020 (2018).

$5 \quad$ Nakano, K. et al. Anatomy of the energetic driving force for charge generation in organic solar cells. Nature Communications 10, 2520, doi:10.1038/s41467-019-10434-3 (2019).

$6 \quad$ Ward, A. J. et al. The Impact of Driving Force on Electron Transfer Rates in Photovoltaic DonorAcceptor Blends. Advanced Materials 27, 2496-2500, doi:10.1002/adma.201405623 (2015).

$7 \quad \mathrm{Li}, \mathrm{Y}$. et al. Non-fullerene acceptor with low energy loss and high external quantum efficiency: towards high performance polymer solar cells. Journal of Materials Chemistry A 4, 5890-5897, doi:10.1039/C6TA00612D (2016). 
Chen, S. et al. A Wide-Bandgap Donor Polymer for Highly Efficient Non-fullerene Organic Solar Cells with a Small Voltage Loss. Journal of the American Chemical Society 139, 6298-6301, doi:10.1021/jacs.7b01606 (2017).

9 Yang, C. et al. Effects of energy-level offset between a donor and acceptor on the photovoltaic performance of non-fullerene organic solar cells. Journal of M aterials Chemistry A 7, 18889-18897, doi:10.1039/C9TA04789A (2019).

10 Zhang, J. et al. Accurate Determination of the Minimum HOMO Offset for Efficient Charge Generation using Organic Semiconducting Alloys. Advanced Energy Materials n/a 1903298, doi:10.1002/aenm.201903298.

11 Scharber, M. C. et al. Design Rules for Donors in Bulk-Heterojunction Solar Cells-Towards $10 \%$ Energy-Conversion Efficiency. Advanced Materials 18, 789-794, doi:10.1002/adma.200501717 (2006).

12 Unger, T. et al. The Impact of Driving Force and Temperature on the Electron Transfer in DonorAcceptor Blend Systems. The Journal of Physical Chemistry C $\mathbf{1 2 1}$ 22739-22752, doi:10.1021/acs.jpcc.7b09213 (2017).

13 Hendriks, K. H., Wijpkema, A. S. G., van Franeker, J. J., Wienk, M. M. \& Janssen, R. A. J. Dichotomous Role of Exciting the Donor or the Acceptor on Charge Generation in Organic Solar Cells. Journal of the American Chemical Society 138, 10026-10031, doi:10.1021/jacs.6b05868 (2016).

14 Coffey, D. C. et al. An Optimal Driving Force for Converting Excitons into Free Carriers in Excitonic Solar Cells. The Journal of Physical Chemistry C 116, 8916-8923, doi:10.1021/.jp302275z (2012).

15 Ohkita, H. et al. Charge Carrier Formation in Polythiophene/Fullerene Blend Films Studied by Transient Absorption Spectroscopy. Journal of the American Chemical Society 130, 3030-3042, doi:10.1021/ja076568q (2008).

16 Li, S. et al. Highly Efficient Fullerene-Free Organic Solar Cells Operate at Near Zero Highest Occupied Molecular Orbital Offsets. Journal of the American Chemical Society 141, 3073-3082, doi:10.1021/jacs.8b12126 (2019).

$17 \mathrm{Li}, \mathrm{Y}$. et al. A fused-ring based electron acceptor for efficient non-fullerene polymer solar cells with small HOMO offset. Nano Energy 27, 430-438, doi:https://doi.org/10.1016/j.nanoen.2016.07.019 (2016).

18 Tereshchenko, I. V. et al. The Role of Semilabile Oxygen Atoms for Intercalation Chemistry of the Metal-Ion Battery Polyanion Cathodes. Journal of the American Chemical Society 140, 3994-4003, doi:10.1021/jacs.7b12644 (2018).

19 Zhang, J. et al. Accurate Determination of the Minimum HOMO Offset for Efficient Charge Generation using Organic Semiconducting Alloys. Advanced Energy Materials 10, 1903298, doi:10.1002/aenm.201903298 (2020).

20 Zhou, Z. et al. Subtle M olecular Tailoring Induces Significant M orphology Optimization Enabling over 16\% Efficiency Organic Solar Cells with Efficient Charge Generation. Advanced Materials 32 , 1906324, doi:10.1002/adma.201906324 (2020).

21 Chen, S. et al. Efficient Nonfullerene Organic Solar Cells with Small Driving Forces for Both Hole and Electron Transfer. Advanced M aterials 30, 1804215, doi:10.1002/adma.201804215 (2018).

22 Yan, C. et al. Non-fullerene acceptors for organic solar cells. Nature Reviews Materials 3, 18003, doi:10.1038/natrevmats.2018.3 (2018).

23 Baran, D. et al. Reduced voltage losses yield $10 \%$ efficient fullerene free organic solar cells with $>1 \mathrm{~V}$ open circuit voltages. Energy \& Environmental Science 9, 3783-3793, doi:10.1039/C6EE02598F (2016).

24 Wan, X., Li, C., Zhang, M. \& Chen, Y. Acceptor-donor-acceptor type molecules for high performance organic photovoltaics - chemistry and mechanism. Chemical Society Reviews, doi:10.1039/D0CS00084A (2020).

25 Sworakowski, J. How accurate are energies of HOMO and LUMO levels in small-molecule organic semiconductors determined from cyclic voltammetry or optical spectroscopy? Synthetic M etals 235, 125-130, doi:https:// doi.org/10.1016/j.synthmet.2017.11.013 (2018).

26 Janssen, R. A. J. \& Nelson, J. Factors Limiting Device Efficiency in Organic Photovoltaics. Advanced Materials 25, 1847-1858, doi:10.1002/adma.201202873 (2013). 
27 Jin, F. et al. Improved Charge Generation via Ultrafast Effective Hole-Transfer in All-Polymer Photovoltaic Blends with Large Highest Occupied Molecular Orbital (HOMO) Energy Offset and Proper Crystal Orientation. Advanced Functional Materials 28, 1801611, doi:10.1002/adfm.201801611 (2018).

28 Aplan, M. P. et al. Revealing the Importance of Energetic and Entropic Contributions to the Driving Force for Charge Photogeneration. ACS Applied Materials \& Interfaces 10, 39933-39941, doi:10.1021/acsami.8b12077 (2018).

29 Perdigón-Toro, L. et al. Barrierless Free Charge Generation in the High-Performance PM 6:Y6 Bulk Heterojunction Non-Fullerene Solar Cell. Advanced Materials 32, 1906763, doi:10.1002/adma.201906763 (2020).

30 Ramirez, I., Causa', M., Zhong, Y., Banerji, N. \& Riede, M. Key tradeoffs limiting the performance of organic photovoltaics. Advanced Energy M aterials 8, Article: 1703551 (2018).

31 Mohapatra, A. A. et al. Förster Resonance Energy Transfer Drives Higher Efficiency in Ternary Blend Organic Solar Cells. ACS Applied Energy Materials 1, 4874-4882, doi:10.1021/acsaem.8b00896 (2018).

32 Gautam, B. R., Younts, R., Carpenter, J., Ade, H. \& Gundogdu, K. The Role of FRET in Non-Fullerene Organic Solar Cells: Implications for M olecular Design. The Journal of Physical Chemistry A 122 37643771, doi:10.1021/acs.jpca.7b12807 (2018).

$33 \mathrm{Bi}$, P. et al. Dual Förster resonance energy transfer effects in non-fullerene ternary organic solar cells with the third component embedded in the donor and acceptor. Journal of M aterials Chemistry A 5, 12120-12130, doi:10.1039/C7TA01557G (2017).

34 Karki, A. et al. Unifying Charge Generation, Recombination, and Extraction in Low-Offset NonFullerene Acceptor Organic Solar Cells. Advanced Energy Materials n/a 2001203, doi:10.1002/aenm.202001203.

35 Karuthedath, S. et al. Impact of Fullerene on the Photophysics of Ternary Small M olecule Organic Solar Cells. Advanced Energy M aterials 9, 1901443, doi:10.1002/aenm.201901443 (2019).

36 Zhong, Y. et al. Sub-picosecond charge-transfer at near-zero driving force in polymer:non-fullerene acceptor blends and bilayers. Nature Communications 11, 833, doi:10.1038/s41467-020-14549-w (2020).

37 Liang, R.-Z. et al. Carrier Transport and Recombination in Efficient "All-Small-Molecule" Solar Cells with the Nonfullerene Acceptor IDTBR. Advanced Energy Materials 8, 1800264, doi:10.1002/aenm.201800264 (2018).

38 Alamoudi, M. A. et al. Impact of Nonfullerene Acceptor Core Structure on the Photophysics and Efficiency of Polymer Solar Cells. ACS Energy Letters 3, 802-811, doi:10.1021/acsenergylett.8b00045 (2018).

39 Yuan, J. et al. Single-Junction Organic Solar Cell with over 15\% Efficiency Using Fused-Ring Acceptor with Electron-Deficient Core. Joule 3, 1140-1151, doi:https://doi.org/10.1016/j.joule.2019.01.004 (2019).

40 Gao, F. A New Acceptor for Highly Efficient Organic Solar Cells. Joule 3, 908-909, doi:https://doi.org/10.1016/j.joule.2019.03.027 (2019).

41 Yoshida, H. Note: Low energy inverse photoemission spectroscopy apparatus. Review of Scientific Instruments 85, 016101, doi:10.1063/1.4860055 (2014).

42 Huifeng, Y. et al. Design and Synthesis of a Low Bandgap Small Molecule Acceptor for Efficient Polymer Solar Cells. Advanced M aterials 28, 8283-8287, doi:doi:10.1002/adma.201602642 (2016).

43 Huang, J.-S. et al. Polymer bulk heterojunction solar cells employing Förster resonance energy transfer. Nature Photonics 7, 479, doi:10.1038/nphoton.2013.82 (2013).

44 Sapsford, K. E., Berti, L. \& Medintz, I. L. Materials for Fluorescence Resonance Energy Transfer Analysis: Beyond Traditional Donor-Acceptor Combinations. Angewandte Chemie International Edition 45, 4562-4589, doi:10.1002/anie.200503873 (2006).

45 Poelking, C. \& Andrienko, D. Design Rules for Organic Donor-Acceptor Heterojunctions: Pathway for Charge Splitting and Detrapping. Journal of the American Chemical Society 137, 6320-6326, doi:10.1021/jacs.5b02130 (2015). 
46 Poelking, C. et al. Impact of mesoscale order on open-circuit voltage in organic solar cells. Nature Materials 14, 434-439, doi:10.1038/nmat4167 (2015).

47 Castet, F., D'Avino, G., M uccioli, L., Cornil, J. \& Beljonne, D. Charge separation energetics at organic heterojunctions: on the role of structural and electrostatic disorder. Physical Chemistry Chemical Physics 16, 20279-20290, doi:10.1039/C4CP01872A (2014).

48 Schwarze, M. et al. Band structure engineering in organic semiconductors. Science 352 1446-1449, doi:10.1126/science.aaf0590 (2016).

49 D'Avino, G. et al. Electrostatic phenomena in organic semiconductors: fundamentals and implications for photovoltaics. Journal of Physics: Condensed Matter 28, 433002, doi:10.1088/09538984/28/43/433002 (2016).

50 Schwarze, $M$. et al. Impact of molecular quadrupole moments on the energy levels at organic heterojunctions. Nature Communications 10, 2466, doi:10.1038/s41467-019-10435-2 (2019).

51 de Sousa, L. E., Coropceanu, V., da Silva Filho, D. A. \& Sini, G. On the Physical Origins of Charge Separation at Donor-Acceptor Interfaces in Organic Solar Cells: Energy Bending versus Energy Disorder. Advanced Theory and Simulations n/a 1900230, doi:10.1002/adts.201900230.

52 Gasparini, N. e. a. Single component organic solar cells based on non-fullerene acceptors. submitted.

53 Liang, R.-Z. et al. Additive-Morphology Interplay and Loss Channels in "All-Small-M olecule" Bulkheterojunction (BHJ) Solar Cells with the Nonfullerene Acceptor IDTTBM. Advanced Functional Materials 28, 1705464, doi:10.1002/adfm.201705464 (2018).

54 Holliday, S. et al. High-efficiency and air-stable P3HT-based polymer solar cells with a new nonfullerene acceptor. Nature Communications 7, 11585, doi:10.1038/ncomms11585 (2016).

55 Frisch, M. J. et al. (Wallingford CT, 2009).

56 Thole, B. T. M olecular polarizabilities calculated with a modified dipole interaction. Chemical Physics 59, 341-350, doi:https://doi.org/10.1016/0301-0104(81)85176-2 (1981).

57 van Duijnen, P. T. \& Swart, M. Molecular and Atomic Polarizabilities: Thole's Model Revisited. The Journal of Physical Chemistry A 102, 2399-2407, doi:10.1021/jp980221f (1998).

58 Stone, A. J. Distributed Multipole Analysis: Stability for Large Basis Sets. Journal of Chemical Theory and Computation 1, 1128-1132, doi:10.1021/ct050190+(2005). 